\title{
Scaling Laws for Heterogeneous Cognitive Radio Networks with Cooperative Secondary Users
}

\author{
Riheng Jia, Jinbei Zhang, Xinbing Wang, Xiaohua Tian \\ Dept. of Electronic Engineering \\ Shanghai Jiao Tong University, China \\ Email: $\{$ jiariheng, abelchina, xwang8, xtian\}@sjtu.edu.cn
}

\author{
Qian Zhang \\ Dept. of Computer Science and Engineering \\ Hong Kong University of Science and Technology, HK \\ Email: qianzh@cse.ust.hk
}

\begin{abstract}
Cognitive radio (CR) technique is considered an effective mechanism to relieve the spectrum scarcity issue, where the secondary users (SUs) can utilize the idle spectrum of the primary users (PUs). How the performance of the wireless network will be influenced by the introduction of CR technique has been attracting much attention in past years. While many efforts have been made to study the cognitive radio network, where the data source and the destination (S-D) is homogeneously distributed, the research on cognitive radio networks (CRN) with heterogeneous S-D distribution is still very limited. In this paper, we investigate the throughput and delay scaling law in the heterogeneous cognitive radio network (HCRN), where the S-D pair follows a rank based model and SUs provide relay service for PUs in reciprocating the utilization of PUs' idle spectrum. By applying a cellular TDMA scheduling scheme, we show that the primary network throughput is the same for different heterogeneous extents of S-D distribution owing to the flexible assistance of SUs, while the throughput of secondary networks is proven to be changing with respect to the S-D heterogeneity exponent denoted by $\alpha$. In addition, the delay scaling are derived for both primary and secondary networks and shown to be altering in accordance with $\alpha$. Further, we reveal that the density of SUs required to assist PUs can be dramatically reduced when considering the S-D heterogeneity, while achieving the same primary network throughput.
\end{abstract}

\section{INTRODUCTION}

Recently, there is more and more stress over the alreadycrowded radio spectrum due to the explosive growth of largescale wireless applications. That motivates people to explore the cognitive radio network (CRN) for its efficient usage of spectrum. As Gupta and Kumar built up the foundation of throughput and delay scaling laws for wireless networks in [1], the researchers started to explore how the fundamental performance scaling law will be affected by the cognitive radio (CR) technique. $\mathrm{Vu}$ et al. considered the throughput scaling law for single-hop cognitive radio networks and obtained a linear scaling of the secondary network in [2], [3]. Jeon et al. studied the throughput scaling of a cognitive network under general environment and showed that both primary and secondary networks can achieve the same throughput scaling law as a stand-alone network while the secondary network may suffer from a finite outage probability in [4]. Yin et al. developed throughput scaling laws under a similar assumption and adopted transmission protocols which could guarantee zero outage probability for secondary networks in [5]. Further, Sun et al. investigated the impact of Blackholes on large- scale CRN [6] and effectively bounded the size of Blackholes through percolation theory.

A common assumption in all the afore-mentioned works of $\mathrm{CRN}$ is that the source and destination (S-D) is homogeneously distributed. However, we consider a more realistic situation where the distribution of S-D is no longer homogeneous, but following a rank based model in accordance with the behavior that source would prefer the closer rather than the further destination to communicate in real life using some locating information [7].

The distribution of $\mathrm{S}-\mathrm{D}$ is developed from the original network architecture [8], [9] to the emerging useful models which capture the characteristic of S-D such as distance based model and rank based model [10], [11]. On foundation of these works, the heterogeneous feature of S-D distribution is brought into wireless networks and their impact on the performance of wireless networks began to draw much attention. Azimdoostv et al. considered wireless social networks with social groups consisting of local contacts as well as long-range contacts and the corresponding throughput had also been studied in [12]. Fu et al. incorporated the rank based model into wireless networks and explored the impact of traffic locality on capacity over the network under different traffic patterns in [13].

We notice that so far, there is still no research on concerning the impact of heterogeneous distribution of S-D on cognitive radio networks. It remains unknown that how the heterogeneity of S-D distribution will influence the performance scaling law of CRN. Gao et al. considered the situation where the secondary users (SUs) are assumed to be willing to share the primary users' (PUs) burden of routing packets for utilizing the idle spectrum of PUs in [14]. Thus, the throughput of primary networks could be improved by the aid of SUs. It is also significant to investigate whether the SUs could still help improving the throughput of primary networks under the heterogeneous setting.

In this paper, we study the throughput and delay of heterogeneous cognitive radio networks (HCRN). The heterogeneous distribution of S-D is generated by employing a rank based model and we use this model to bound the distance between source and destination (S-D distance). Then we propose cooperative routing protocols to fully utilize the heterogeneity of S-D distribution and deeply explore how the new network model will make an impact on the scaling law performance. 
In addition, the cost of network deployment issue is also discussed. Our main contributions are summarized as follows:

- We show that the heterogeneous distribution of S-D will not influence the PUs throughput scaling and the primary network can achieve the near optimal per-node throughput of $\Theta\left(\frac{1}{\log n}\right)$ in whatever ranges of $\alpha$, where $\alpha$ is the S$\mathrm{D}$ heterogeneity exponent. Besides, we demonstrate that the delay of primary networks differs in different ranges of $\alpha$, which is affected by the heterogeneity of S-D distribution.

- The throughput and delay scaling law of the secondary network in HCRN are also investigated. It is testified that the throughput and delay of secondary networks will alter in synchronization with the range of $\alpha$. Both the near optimal delay and throughput can be achieved in certain ranges of $\alpha$. In addition, we prove that the secondary network can achieve the same scaling law of delay and throughput as being a stand-alone network, which means providing PUs help will not degrade the performance of the secondary network.

- A distinguishing characteristic in our paper is that the density of SUs can be different for diverse heterogeneous extents of S-D distribution, while guaranteeing the near optimal per-node throughput of primary networks. We show that our results are more general compared with that in [14]. In this situation, we could appropriately arrange the number of SUs and make the network deployment much more economical.

The rest of this paper is organized as follows. In Section II, the system model is defined. We study traffic locality under the rank based model in Section III. In Section IV, throughput and delay scaling laws of HCRN are derived. In Section V, we discuss the results. Our paper is concluded in Section VI.

\section{SySTEM Model}

\section{A. Network Geometry}

The network extension is considered to be an unit square where primary and secondary nodes coexist. The primary and secondary nodes are randomly distributed according to a Poisson Point Process (P.P.P.) of density $n$ and $m$, respectively. The nodes of the two networks are grouped into one to one source-destination (S-D) pairs upon the rank based model. $n$ and $m$ are correlated with

$$
m=n^{\beta},
$$

where $\beta$ may change in different situations which we will show the details later in Section IV. But $\beta>1$ is guaranteed, thus the density of the primary nodes is higher than that of the secondary nodes.

\section{B. Transmission Model}

For the wireless channel in our work, shadowing or small scale fading issues are ignored and we only consider the path loss for simplicity. Therefore, the channel power gain is given as

$$
g(d)=d^{-\gamma},
$$

where $d$ is the distance between a transmitter and its receiver, $\gamma>2$ indicates the path-loss exponent.
We assume that each transmission of primary and secondary networks deploys a scheme that can achieve the additive white gaussian noise (AWGN) channel capacity which is given by the well known formula $R=\log (1+S N R)$. Specifically, the $i$-th primary Tx-Rx pair can communicate at a rate of

$$
R_{p}^{i}=\log \left(1+\frac{P_{p}^{i} g\left(\left\|X_{p, t x}^{i}-X_{p, r x}^{i}\right\|\right)}{N_{0}+I_{p}^{i}+I_{s p}^{i}}\right),
$$

where $\|$.$\| represents the Euclidean distance between arbitrary$ two nodes in the unit square. $I_{p}^{i}$ is the sum interference from all the other concurrent primary transmitters to the receiver of the $i$-th primary pair. $I_{s p}^{i}$ is the sum interference from all the concurrent secondary transmitters to the receiver of the $i$-th primary pair. Suppose that $N_{p}$ primary pairs and $N_{s}$ secondary pairs communicate simultaneously, $I_{p}^{i}$ and $I_{s p}^{i}$ are given by

$$
\begin{aligned}
& I_{p}^{i}=\sum_{k=1, k \neq i}^{N_{p}} P_{p}^{k} g\left(\left\|X_{p, t x}^{k}-X_{p, r x}^{i}\right\|\right), \\
& I_{s p}^{i}=\sum_{k=1}^{N_{s}} P_{s}^{k} g\left(\left\|X_{s, t x}^{k}-X_{p, r x}^{i}\right\|\right) .
\end{aligned}
$$

Similarly, the $j$-th secondary pair can communicate at a rate of

$$
R_{s}^{j}=\log \left(1+\frac{P_{s}^{j} g\left(\left\|X_{s, t x}^{j}-X_{s, r x}^{j}\right\|\right)}{N_{0}+I_{s}^{j}+I_{p s}^{j}}\right),
$$

where $I_{s}^{j}$ and $I_{p s}^{j}$ are similarly defined as that in Equation (4).

\section{Rank Based Model}

In this paper, the heterogeneous distribution of S-D is investigated to capture the relationship between source and destination in the real world. More specifically, we adopt a rank based model where the probability of any source node finding a designated destination node is inversely proportional to the $\alpha$-th power of the number of closer nodes. This distinguishes our work with most of the previous analysis regarding throughput and delay scaling laws of general cognitive radio networks since the S-D distance is no longer bounded as $\Theta(1)$, and the situation would become much more complicated.

For simplicity, considering a single network with $n$ nodes randomly distributed in a given area. Picking out arbitrary two nodes $i$ and $j$, then define the rank of $j$ with respect to $i$ as

$$
\operatorname{Rank}_{i}(j)=\left|\left\{k:\left\|X_{i}-X_{k}\right\|<\left\|X_{i}-X_{j}\right\|\right\}\right|,
$$

where $X_{i}$ and $X_{j}$ denote the location of node $i$ and $j$ in a single network, respectively. Then the probability that $j$ is the destination of $i$ is modeled as

$$
\mathbb{P}(i \rightarrow j) \propto \frac{1}{\operatorname{Rank}_{i}^{\alpha}(j)},
$$

where $\alpha$ is the S-D heterogeneity exponent and $\alpha \geq 0$, denoting for short $H_{n}=\sum_{j=1}^{n} 1 / j^{\alpha}$ (suppose there are $n$ nodes in the network), the distribution law is

$$
\mathbb{P}(i \rightarrow j)=\frac{1}{H_{n} \operatorname{Rank}_{i}^{\alpha}(j)} .
$$




\section{Throughput and Delay}

The per-node throughput of a S-D pair is defined as the average data rate that each source node can transmit to its chosen destination, which is asymptotically determined by the network density. In the following, we use $\lambda_{p}(n)$ and $\lambda_{s}(m)$ to denote the per-node throughput for primary and secondary networks, respectively.

In brief, we use a fluid model [15] for delay analysis and the delay is defined as the average time slots a packet takes to be delivered from source to destination. We use $D_{p}(n)$ and $D_{s}(m)$ to denote packet delays for the primary and secondary networks, respectively.

\section{TRAFFIC LOCALITY}

It is notable that we adopt a rank based model to determine the destination for each primary or secondary source node in heterogeneous cognitive radio networks. This makes the traffic pattern significantly different from the traditional cognitive radio network. According to Equation (8), a source node tends to choose a closer node as its destination and therefore resulting in a certain degree of traffic locality.

Some useful properties of rank based model had been established in [13]. We refer readers to [13] for details and just provide the result here,

Lemma 1. Considering a single network with $n+1$ nodes and denote $Y_{i}$ as the destination selected by the $i$-th node $X_{i}$, Then the average $S-D$ distance $\mathbb{E}\left(\left\|X_{i}-Y_{i}\right\|\right)$ in a unit square can be bounded as:

$$
\begin{aligned}
\mathbb{E}\left(\left\|X_{i}-Y_{i}\right\|\right) & =\mathbb{E}_{r}\left(\mathbb{E}\left(\left\|X_{i}-Y_{i}\right\| \mid r: n\right)\right) \\
& \sim \frac{1}{H_{n} \sqrt{n}} \sum_{r=1}^{n} \frac{\sqrt{r}}{r^{\alpha}} \\
& \sim\left\{\begin{array}{lr}
1 / \sqrt{n} & \alpha>3 / 2 \\
\log n / \sqrt{n} & \alpha=3 / 2 \\
n^{1-\alpha} & 1<\alpha<3 / 2 \\
1 / \log n & \alpha=1 \\
1 & 0 \leq \alpha<1,
\end{array}\right.
\end{aligned}
$$

where $\alpha$ denotes the S-D heterogeneity exponent. It can be seen in Equation (9) that the average S-D distance varies with respect to $\alpha$ and such results will inevitably cause the complexity in our network model and bring challenge to the calculation of throughput and delay in HCRN.

\section{Throughrut and Delay Scaling Laws of Heterogeneous Cognitive Radio Networks}

We notice that the distribution of S-D is much more complicated compared with that in [4], [5]. In our model, the average S-D distance could not be easily bounded as $\Theta(1)$. We are interested in how the achievable throughput and delay alter with respect to $\alpha$. We also wonder whether the throughput and delay of primary networks could be improved by the aid of SUs under the new circumstances. An interesting case is that when $\alpha$ increases beyond 3/2, the transmission tends to be a

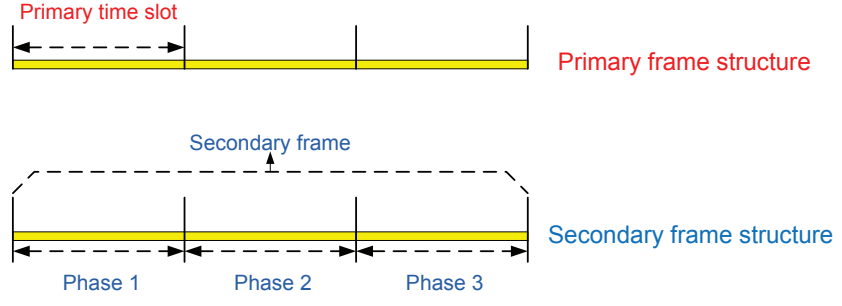

Fig. 1: Frame relationship between primary and secondary networks.

single-hop fashion. Furthermore, we prove that the density of SUs required to assist PUs can be dramatically reduced while achieving the same primary network throughput.

In this section, we will first introduce the transmission protocols for both primary and secondary networks and briefly analyze the corresponding interference. Then we mainly focus on the achievable throughput and delay of both networks in HCRN.

\section{A. Primary Protocol}

- The unit square is divided into small-square primary cells with size $a_{p}(n)$. The condition of $a_{p}(n) \geq \frac{\sqrt{2} \beta \log n}{n}$ is derived to guarantee the full connectivity within the primary network and thus each cell has at least one primary node w.h.p..

- Every 64 primary cells are supposed to grouped into a primary cluster and we use a round-robin fashion to determine when the cells could be active in each primary cluster. The transmission time is divided into TDMA frames where each frame has 64 primary time slots that correspond to the number of primary cells in each primary cluster. Note that we make every primary cluster have 64 primary cells, thus enabling us arrange the preservation and recycling regions much more conveniently.

- Define the horizontal data path (HDP) and the vertical data path (VDP) of a S-D pair as the horizon line and the vertical line connecting a source to its destination, respectively. Each source transmits data to its destination by first hopping to the adjacent cells on its HDP and then the VDP. The detailed transmission process is explained in [5] due to page limit.

- When a primary cell is active, each primary source node transmit its own packets in order. Afterwards, each of the S-D paths passing through the cell could be served by the designated relay node for one packet only. Note that when a primary source node finished sending out its own packets, it could be a designated relay node either. Each primary time slot is divided into packet slots according to the fluid model. For each packet, it will be handed over directly to the destination if the destination node happens to be in the adjacent cell. Otherwise, the packets are blindly broadcasted to the nodes in the adjacent cell. At each packet transmission, we denote the transmitting power by $P a_{p}^{\frac{\gamma}{2}}(n)$, where $P$ is a constant.

- Note that, the above primary protocol is operating independently of whether the secondary network is present or not. We will show later that the primary network can achieve better 


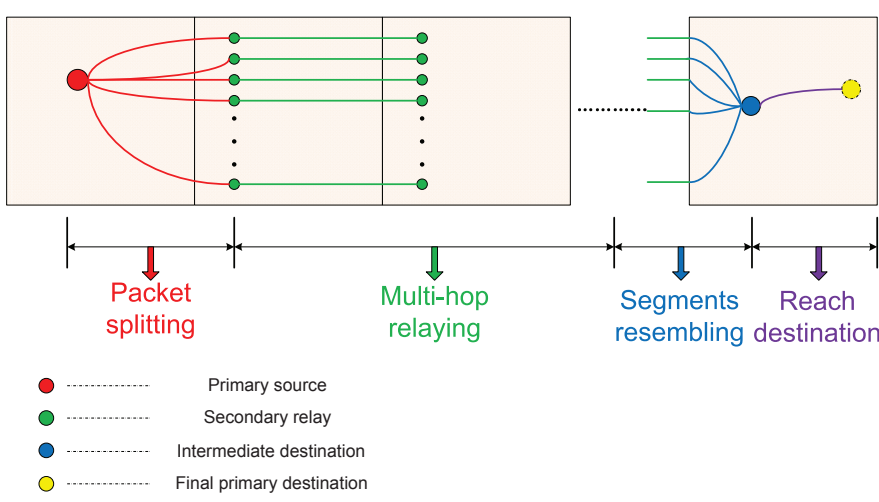

Fig. 2: The process of primary packet transmission.

performance than being a stand-alone network in [1] with the aid of secondary users.

\section{B. Secondary Protocol}

We assume that the secondary nodes are capable of pretending to be the primary nodes due to the so called "softwareprogrammability". So the "fake" primary nodes could be chosen to help primary users to relay packets. Once a secondary node is picked up for relaying packets, it will keep silent unless its related primary cell becomes active.

As we will discuss later that the time-sharing strategy will only incur a constant penalty on the achievable throughput and delay of secondary networks. Thus we divide each secondary frame into three equal-length phases, thus each of them equals to one primary time slot which is illustrated in Fig.1.

Similarly as defined in the primary protocol, the unit area is divided into small-square secondary cells with size $a_{s}(m)$. We have to ensure $a_{s}(m) \geq \frac{2 \log m}{m}$ to maintain the full connectivity within the secondary network. The secondary cells are grouped into secondary clusters with each secondary cluster of 64 secondary cells. Accordingly, each secondary cluster follows a 64-TDMA pattern to communicate. A preservation region consisting of nine primary cells and a layer of secondary cells is defined in our paper. Each particular active primary cell is supposed to be centered at the preservation region. Only the secondary transmitters in an active secondary cell outside all the preservation regions can transmit data, otherwise, the data has to be temporarily stored in buffer until the preservation region is clear.

Now we show the details about the three phases as follows:

Phase 1: When an active secondary cell is outside the preservation regions, each secondary source node is allowed to transmit one own packet and then serve each S-D path passing through the cell. The routing of secondary packets is similar to that defined in the primary protocol. At each packet transmission, the transmitting power is denoted by $P a_{s}^{\frac{\gamma}{2}}(m)$.

Phase 2 : Only the secondary nodes who buffered the primary packets are permitted to access the spectrum. We notice that the throughput per secondary S-D pair is less than that of primary S-D pair which is caused by the different node densities, so packet splitting is needed to prevent the bottleneck effect from happening in relaying primary packets through the secondary network. As shown in Fig.2, suppose primary source node pours all the split primary segments to the neighboring cell, then in each primary cell, we assume that there are $N$ secondary nodes including the designated relay node who are preparing to relay along the data paths passing through the cell. Each secondary node is assigned to relay $1 / N$ portion of the primary packet to the intermediate destination by using a multi-hop scheme. Due to traffic locality, the value of $N$ may vary in different ranges of $\alpha$, which is much more complicated compared with that in [14]. Based on different traffic patterns, the value of $N$ is set as follows:

$$
N= \begin{cases}\Theta(\sqrt{\log n}) & \alpha=\frac{3}{2}, \beta>1 \\ \Theta\left(n^{\left(\frac{3}{2}-\alpha\right) \beta} / \sqrt{\log n}\right) & 1<\alpha<\frac{3}{2}, \beta \geq \frac{1}{\alpha-\frac{1}{2}} \\ \Theta\left(n^{\frac{\beta}{2}} / \log ^{\frac{3}{2}} n\right) & \alpha=1, \beta \geq 2 \\ \Theta\left(n^{\frac{\beta}{2}} / \sqrt{\log n}\right) & 0 \leq \alpha<1, \beta \geq 2 .\end{cases}
$$

We can easily find that the heterogeneous distribution of S-D do make an impact on the number of SUs required to help relaying the primary packets from the equation above. It should point out that the range of $\beta$ here is to guarantee that there are more than $N$ secondary users in each primary cell. When $0 \leq \alpha \leq 1$, we can calculate the range of $\beta \geq 2$, which is the same result as that in [14]. But when $1<\alpha<\frac{3}{2}$ or $\alpha=\frac{3}{2}$, the results turn to be $\beta \geq \frac{1}{\alpha-\frac{1}{2}}$ or $\beta>1$, respectively, which means the node density of secondary networks can be sharply reduced compared with that in [14] and thus the cost of network deployment can be reduced in practical. The situation where $\alpha>\frac{3}{2}$ is ignored in Equation (10) as the transmission tends to be a single-hop fashion which we will show the details in the next subsection. The transmission scheme is similar as that in the first phase.

Phase 3 : A recycling region consisted by nine primary cells and a layer of secondary cells around them is defined as to recycle the primary packet from the intermediate destination. Noting that each recycling region is located between two preservation regions and they are not overlapped with each other. The recycling process is assumed to happen in the center cell of the recycling region. At each packet transmission, the transmitting power of the intermediate destination node is the same as that of primary node.

\section{Throughput Analysis}

Before we proceed to analyze the per-node throughput of primary and secondary networks, we first consider the transmission mode over the network in term of the average hops for each packet to go through. We denote $\mathbb{E}_{h}$ as the average number of hops per packet for a single network, then

$$
\mathbb{E}_{h} \sim \frac{\mathbb{E}\left(\left\|X_{i}-Y_{i}\right\|\right)}{\sqrt{a}},
$$

where $a$ denotes the area of each cell in the network. For simplicity, we take the transmission of primary networks as an example to show how $\mathbb{E}_{h}$ changes with respect to $\alpha$. 
By definition, in primary networks we have

$$
\begin{aligned}
\mathbb{E}_{h} \sim & \frac{\mathbb{E}\left(\left\|X_{p}^{i}-Y_{p}^{i}\right\|\right)}{\sqrt{a_{p}(n)}} \\
& \sim\left\{\begin{array}{lr}
1 / \sqrt{\log n} & \alpha>3 / 2 \\
\sqrt{\log n} & \alpha=3 / 2 \\
n^{\frac{3}{2}-\alpha} / \sqrt{\log n} & 1<\alpha<3 / 2 \\
\sqrt{n / \log ^{3} n} & \alpha=1 \\
\sqrt{n / \log n} & 0 \leq \alpha<1 .
\end{array}\right.
\end{aligned}
$$

It is easy to see that $\mathbb{E}_{h} \rightarrow \infty$ when $0 \leq \alpha \leq \frac{3}{2}$, and $\mathbb{E}_{h} \rightarrow 0$ when $\alpha>\frac{3}{2}$. It means that when $0 \leq \alpha \leq \frac{3}{2}$, we can still use our cellular TDMA multi-hop scheme to analyze per-node throughput of the primary and secondary network. However when the value of $\alpha$ exceeds $\frac{3}{2}$, a particular S-D pair is within the cell in which it is located and it tends to be a single-hop transmission. In this subsection, we mainly deal with the throughput using multi-hop scheme and will present the single-hop scheme when $\alpha>\frac{3}{2}$ later. In the rest of this paper, we denote $\mathbb{E}\left(\left\|X_{p}^{i}-Y_{p}^{i}\right\|\right)$ as $E_{s d}(n)$ for short. Similarly, $\mathbb{E}\left(\left\|X_{s}^{i}-Y_{s}^{i}\right\|\right)$ is denoted as $E_{s d}(m)$.

1) Primary Network: In order to obtain the throughput scaling law, we first give the following lemmas.

Lemma 2. The probability that a randomly selected designated relay node is a secondary node in a primary cell approaches one w.h.p..

Proof: Denoting the probability by $\gamma$, from the proof of Theorem 1, we know that in a primary cell, the number of primary nodes is $\Theta\left(n a_{p}(n)\right)$ and the number of secondary nodes is $\Theta\left(m a_{p}(n)\right)$. Then the probability can be expressed as $\gamma=\frac{\Theta\left(m a_{p}(n)\right)}{\Theta\left(m a_{p}(n)\right)+\Theta\left(n a_{p}(n)\right)}$. More specifically, $\gamma=\frac{1}{1+n^{1-\beta}}$, which approaches one as $n \rightarrow \infty$, where $\beta>1$.

Lemma 3. With the primary protocol defined previously, an active primary cell can support a constant data rate of $L_{1}$, the intermediate destination node can deliver the primary packets to the intended primary destination at a constant rate of $L_{2}$, where $L_{1}, L_{2}>0$ and are independent of $n$ and $m$.

We refer readers to [14] for detailed proof of Lemma 3.

Based on Lemma 2 and Lemma 3, we have the following theorem.

Theorem 1. With the protocols given previously, the primary network can achieve the following per-node throughput w.h.p.:

$$
\lambda_{p}(n)= \begin{cases}\Theta\left(\frac{1}{n a_{p}(n)}\right) & 0 \leq \alpha \leq 1, \beta \geq 2 \\ \Theta\left(\frac{1}{n a_{p}(n)}\right) & 1<\alpha<\frac{3}{2}, \beta \geq \frac{1}{\alpha-\frac{1}{2}} \\ \Theta\left(\frac{1}{n a_{p}(n)}\right) & \alpha=\frac{3}{2}, \beta>1,\end{cases}
$$

where $a_{p}(n) \geq \sqrt{2} \beta \log n / n$ and $a_{p}(n)=o(1)$.

Proof: In this proof, an upper-bound of the throughput per S-D pair is firstly derived and then we provide a lower-bound by using the secondary protocol in Subsection B.

From Lemma 3, we know that the primary source node can transmit all its packets to the secondary network with a constant data rate of $L_{1}$. Similarly, the primary destination node can receive all its packets with a constant data rate of $L_{2}$. Since only primary source nodes transmit packets in each active primary cell while the secondary relay nodes keep silent. As the size of a primary cell equals to $a_{p}(n)$ and the node density of PUs is $n$, we can easily get that the number of primary source nodes in each primary cell is of $\Theta\left(n a_{p}(n)\right)$ w.h.p., then the throughput per S-D pair is upper-bounded by $\Theta\left(\min \left(\frac{L_{1}}{n a_{p}(n)}, \frac{L_{2}}{n a_{p}(n)}\right)\right)=\Theta\left(\frac{1}{n a_{p}(n)}\right)$.

As to the lower-bound, we calculate the achievable throughput per S-D pair. In the analysis of secondary protocol, each primary source node transmits all its packets to the secondary network w.h.p. (based on Lemma 2) by dividing data into $N$ secondary data paths, each of them at a rate of $\Theta\left(\frac{1}{m E_{s d}(m) \sqrt{a_{s}(m)}}\right)$ given in Equation (18). By setting proper $\sqrt{a_{s}(m)}$ corresponding to different traffic patterns, which satisfy $a_{s}(m) \geq 2 \log m / m$. Thus, a throughput scaling law of $N \Theta\left(\frac{1}{m_{E_{s d}}(m) \sqrt{a_{s}(m)}}\right)=\Theta\left(\frac{1}{n a_{p}(n)}\right)$ can be achieved for each primary source node, which is considered as the lower-bound.

By combining the two bounds, Equation (14) is derived and thus completing the proof.

Noting that the condition of $\beta \geq 2, \beta \geq \frac{1}{\alpha-\frac{1}{2}}$ and $\beta>1$ corresponding to different ranges of $\alpha$ are needed in the proof to guarantee that there are more than $N$ secondary nodes in each primary cell w.h.p.. By setting $a_{p}(n)=\sqrt{2} \beta \log n / n$, the primary network can achieve the following per-node throughput w.h.p.:

$$
\lambda_{p}(n)= \begin{cases}\Theta\left(\frac{1}{\log n}\right) & 0 \leq \alpha \leq 1, \beta \geq 2 \\ \Theta\left(\frac{1}{\log n}\right) & 1<\alpha<\frac{3}{2}, \beta \geq \frac{1}{\alpha-\frac{1}{2}} \\ \Theta\left(\frac{1}{\log n}\right) & \alpha=\frac{3}{2}, \beta>1 .\end{cases}
$$

From Equation (15) we can see that the per-node throughput scaling law of primary networks can be improved from $\Theta(1 / \sqrt{n \log n})$ as in the stand-alone network to $\Theta(1 / \log n)$ with the help of secondary users. We can also find that the density of secondary networks can be sharply reduced to $\beta \geq \frac{1}{\alpha-\frac{1}{2}}\left(1<\alpha<\frac{3}{2}\right)$ or $\beta>1\left(\alpha=\frac{3}{2}\right)$ compared with the results $\beta \geq 2$ in [14], while achieving the same throughput scaling within the primary network.

2) Secondary Network: Using the similar way as the analysis of Equation (13), we can easily get that the transmission also tends to be a single-hop fashion in the secondary network when $\alpha>\frac{3}{2}$, which we will show the details later. In the following, we present the throughput scaling law for the secondary network in a multi-hop fashion.

In order to obtain the throughput scaling law, we first give the following lemmas. 
Lemma 4. Let $n_{\text {st }}$ denote the number of total secondary nodes in the unit square, then we have $\frac{m}{2}<n_{\text {st }}<$ em w.h.p..

The proof can be found in [5].

Lemma 5. With the secondary protocol defined previously, each TX in a secondary cell can support a constant data rate of $K$ which is independent of $m$.

Proof: Since the 64-TDMA scheme and the preservation region are adopted, the interference caused by the other concurrent primary or secondary transmitters could be bounded by a constant. This completes the proof.

We note that the interference at a particular receiver only depends on the scheduling scheme in this situation since all of the interference comes from the concurrent transmitters. Therefore we can use the same method to bound the interference although the S-D distance in our model is different from that in [5].

The next lemma shows the bound of the average number of S-D paths that pass through or originate from each secondary cell.

Lemma 6. The number of secondary $S$-D paths (including both HDPs and VDPs) passing through or originating from each secondary cell is bounded as $\Theta\left(m E_{s d}(m) \sqrt{a_{s}(m)}\right)$ w.h.p., for $o \leq \alpha \leq \frac{3}{2}$.

Proof: According to Lemma 1 in Section III, the distance of secondary S-D pair is bounded by $\Theta\left(E_{s d}(m)\right)$ w.h.p., so it is intuitive that the corresponding HDP (or VDP) has a distance of the same order.

Based on this corollary, we affirm that all the secondary sources of HDPs that passing through or originating from a particular cell should be located in an area of size $\Theta\left(E_{s d}(m) \times\right.$ $\left.\sqrt{a_{s}(m)}\right)$ w.h.p.. We assume $\Theta\left(E_{s d}(m) \times \sqrt{a_{s}(m)}\right)$ could be bounded by $C_{1} E_{s d}(m) \times \sqrt{a_{s}(m)}$ where $C_{1}$ is a constant independent of $m$. We proceed the proof following the Lemma 3 in [4].

Let $m_{h}$ denote the number of HDPs passing through or originating from each secondary cell, by assuming all secondary nodes are sources, the resulting upper bound on $m_{h}$ follows Poisson distribution $\left(\lambda=m \times C_{1} E_{s d}(m) \sqrt{a_{s}(m)}\right)$. Using the well known inequality $\mathbb{P}(X \geq x) \leq \frac{e^{-\lambda}(e \lambda)^{x}}{x^{x}}[16]$, for $x>\lambda$, where $X$ is a Poisson random variable with parameter $\lambda$, we obtain

$$
\begin{aligned}
\mathbb{P}\left(m_{h} \geq 2 \lambda\right) & \leq\left.\frac{e^{-\lambda}(e \lambda)^{x}}{x^{x}}\right|_{x=2 \lambda, \lambda=m c_{1} E_{s d}(m) \sqrt{a_{s}(m)}} \\
& =\left(\frac{e}{4}\right)^{m c_{1} E_{s d}(m) \sqrt{a_{s}(m)}} .
\end{aligned}
$$

Similarly, we denote $m_{v}$ as the number of corresponding VDPs, and we also have

$$
\begin{aligned}
\mathbb{P}\left(m_{v} \geq 2 \lambda\right) & \leq\left.\frac{e^{-\lambda}(e \lambda)^{x}}{x^{x}}\right|_{x=2 \lambda, \lambda=m c_{1} E_{s d}(m) \sqrt{a_{s}(m)}} \\
& =\left(\frac{e}{4}\right)^{m c_{1} E_{s d}(m) \sqrt{a_{s}(m)}} .
\end{aligned}
$$

From Inequality (16) and Inequality (17), we have

$$
\begin{aligned}
\mathbb{P}\left(m_{h}+m_{v} \geq 4 \lambda\right) & \leq\left. 2 \frac{e^{-\lambda}(e \lambda)^{x}}{x^{x}}\right|_{x=2 \lambda, \lambda=m c_{1} E_{s d}(m) \sqrt{a_{s}(m)}} \\
& =2\left(\frac{e}{4}\right)^{m c_{1} E_{s d}(m) \sqrt{a_{s}(m)}},
\end{aligned}
$$

where the last inequality holds due to the union bound.

Therefore the probability that there is at least one cel1 supporting more than $4 \lambda$ data paths is upper bounded by $2 m\left(\frac{e}{4}\right)^{m c_{1} E_{s d}(m) \sqrt{a_{s}(m)}}$ since there are at most $m$ cells in the network. It is not difficult to prove that $2 m\left(\frac{e}{4}\right)^{m c_{1} E_{s d}(m) \sqrt{a_{s}(m)}} \rightarrow 0$ w.h.p., when $\mathbb{E}_{s d}(m)$ varies according to Equation (9) for $0 \leq \alpha \leq \frac{3}{2}$. We just show the proof for the case when $\alpha=\frac{3}{2}$, Other cases could be proved in a similar way.

$$
\begin{aligned}
& \left.2 m\left(\frac{e}{4}\right)^{\lambda=m c_{1} E_{s d}(m) \sqrt{a_{s}(m)}}\right|_{\alpha=\frac{3}{2}}=2 m\left(\frac{e}{4}\right)^{m c_{1} \frac{\log m}{\sqrt{m}} \sqrt{\frac{2 \log m}{m}}} \\
& =2 m\left(\frac{e}{4}\right)^{c_{2} \log ^{3 / 2} m} \\
& <2 m e^{-\left(c_{2} \log ^{3 / 2} m\right) / 8} \\
& =2 m^{1-c_{3} \sqrt{\log m}} \rightarrow 0 \text {. }
\end{aligned}
$$

which completes the proof.

Note that Lemma 6 is new result for the heterogeneous cognitive radio network setup. Based on Lemma 4-6, we have the following theorem.

Theorem 2. With the predefined model and secondary protocol, the secondary network can achieve the following per-node throughput w.h.p.:

$$
\lambda_{s}(m)= \begin{cases}\Theta\left(1 / \log ^{\frac{3}{2}} m\right) & \alpha=\frac{3}{2} \\ \Theta\left(m^{\alpha-\frac{3}{2}} / \sqrt{\log m}\right) & 1<\alpha<\frac{3}{2} \\ \Theta(\sqrt{\log m} / \sqrt{m}) & \alpha=1 \\ \Theta(1 / \sqrt{m \log m}) & 0 \leq \alpha<1\end{cases}
$$

Proof: From Lemma 5 we know that a given TX node in a secondary cell can support a constant data rate of at least $K$, so each secondary S-D pair can achieve a data rate of at least $K$ divided by the maximum number of data paths that pass through or originate from the secondary cell. We already proved that the number of data paths handled by a particular secondary cell is $\Theta\left(m E_{s d}(m) \sqrt{a_{s}(m)}\right)$ w.h.p.. Therefore the achievable per-node throughput is of order $\Theta\left(\frac{1}{m E_{s d}(m) \sqrt{a_{s}(m)}}\right)$ w.h.p.. This completes the proof.

It should point out that since the given time sharing strategy only incurs a constant penalty (i.e., 1/3) on the achievable throughput and delay within the secondary network and thus will not hurt the scaling law performance of secondary networks. Therefore, Theorem 2 still holds when the secondary network operates as a stand-alone network.

3) Throughput Analysis for Single-hop Fashion: As mentioned before, when $\alpha>\frac{3}{2}$, each S-D pair locates within a particular cell w.h.p. for both primary and secondary networks. In this case, we could easily bound the per-node throughput 
of primary and secondary networks by adapting the routing scheme to a single-hop fashion.

We have studied the interference for primary and secondary networks in a cellular TDMA model. Since we remain the scheduling scheme unchanged, Lemma 3 and Lemma 5 still hold in this case. Therefore the S-D pair in both networks can transmit data at a constant rate. We give the achievable per-node throughput of the single-hop fashion through the following theorem.

Theorem 3. In our proposed single-hop scheme, the primary and secondary network can achieve the following per-node throughput w.h.p.:

$$
\lambda_{p}(n)=\Theta\left(\frac{1}{\log n}\right), \lambda_{s}(m)=\Theta\left(\frac{1}{\log m}\right) .
$$

Proof: Since we adopt a single-hop scheme, each S-D pair can transmit data within a particular cell at a constant rate according to Lemma 3 and Lemma 5. Moreover, as stated in the proof of Theorem 1, there are at most $\Theta(\log n)$ nodes in a primary cell and $\Theta(\log m)$ nodes in a secondary cell. Therefore, the achievable throughput is at least the transmission rate divided by the number of nodes in a particular cell for both primary and secondary networks. This completes the proof.

\section{Delay Analysis}

Similar to the throughput analysis, first, we consider the situation when $0 \leq \alpha \leq \frac{3}{2}$. Then we focus on the single-hop fashion, where $\alpha>\frac{3}{2}$.

1) Primary Network: First of all, we calculate the traveling time for the $N$ segments of a primary packet to reach the corresponding intermediate destination node within the secondary network. Since the data paths for the $N$ segments are along the route and an active secondary cell (outside all the preservation regions) transmits one packet for each data path passing through it within a secondary time slot. Assuming that there exists a central entity to coordinate the transmission of $N$ packet segments, thus we can guarantee that the $N$ segments depart from the $N$ nodes, move hop by hop along the data paths, and finally reach the corresponding intermediate destination in a synchronized fashion. According to the fluid model, the $N$ segments experience the same delay in Equation (23).

Let $T_{p}$ and $T_{s}$ denote the durations of the primary and secondary time slot, respectively. According to the proposed protocols, we have $T_{p}=64 T_{s}$. Since each secondary time frame is split into three phases and one of them is used for relaying the primary packet, thus each primary packet suffers from the following delay:

$$
D_{p}(n)=\frac{3}{64} D_{s}(m)+C_{1}+C_{2}=\Theta\left(\frac{E_{s d}(m)}{\sqrt{a_{s}(m)}}\right),
$$

where the secondary network delay $D_{s}(m)$ is defined in Theorem 5. $C_{1}$ denotes the average time for a primary packet traveling from the primary source node to the $N$ secondary relay nodes, $C_{2}$ denotes the average time for a primary packet traveling from the intermediate destination node to the final destination node, which $C_{1}$ and $C_{2}$ are both constants. In order to obtain a better delay performance, we should make $a_{s}(m)$ as large as we could. However, a larger $a_{s}(m)$ results in a decreased throughput per S-D pair in the secondary network and consequently a decreased throughput for the primary network, for the primary traffic traverse over the secondary network w.h.p.

Before proceeding, we give the following lemma.

Lemma 7. The relationship between $a_{p}(n)$ and $a_{s}(m)$ based on the predefined protocols is as follows:

$$
a_{s}(m)=\left(\frac{N n a_{p}(n)}{m E_{s d}(m)}\right)^{2} .
$$

Proof: According to Theorem 1, the maximum throughput per S-D pair for the primary network is $\Theta\left(\frac{1}{n a_{p}(n)}\right)$. Since a primary packet is divided into $N$ segments and then routed by $N$ parallel S-D paths within the secondary network, the supported rate for each secondary S-D pair is required to be $\Theta\left(\frac{1}{N} \times \frac{1}{n a_{p}(n)}\right)$. And based on Theorem 2, the corresponding secondary cell size needs to be set as $\left(\frac{N n a_{p}(n)}{m E_{s d}(m)}\right)^{2}$, where we have $a_{s}(m) \geq 2 \log m / m$ when $a_{p}(n) \geq \sqrt{2} \beta \log n / n$.

Substituting Equation (21) into Equation (20), we have the following theorem.

Theorem 4. Based on the proposed protocols in Subsection A and $B$, the primary network can achieve the following delay w.h.p.

$$
D_{p}(n)=\Theta\left(\frac{n^{\beta-1} E_{s d}(m)}{N a_{p}(n)}\right) .
$$

2) Secondary Network: The delay performance of the secondary network is given by the following theorem.

Theorem 5. Based on the predefined secondary protocol, the delay is given by

$$
D_{s}(m)= \begin{cases}\Theta(\sqrt{\log m}) & \alpha=\frac{3}{2} \\ \Theta\left(m^{\frac{3}{2}-\alpha} / \sqrt{\log m}\right) & 1<\alpha<\frac{3}{2} \\ \Theta\left(\sqrt{m} / \log ^{\frac{3}{2}} m\right) & \alpha=1 \\ \Theta(\sqrt{m} / \sqrt{\log m}) & 0 \leq \alpha<1 .\end{cases}
$$

Proof: We first calculate the average number of hops for each packet to reach the destination along the secondary S-D path. Then we use the fact that each hop costs one secondary packet a constant time to derive the average delay for each secondary S-D pair.

We denote $E_{h}^{s}$ as the average number of hops for each secondary S-D pair, then we have $E_{h}^{s} \sim \frac{E_{s d}(m)}{\sqrt{a_{s}(m)}}$.

Since we use the fluid model, the packet size of each secondary transmission scales proportionally to the throughput $\lambda_{s}(m)$, each packet arriving at a secondary cell will be sent 
to the next active time slot of the cell. It is clear that, w.h.p.,

$$
D_{s}(m)=\frac{2}{n_{s t}} \sum_{j=1}^{\frac{n_{s t}}{2}} \frac{\left\|X_{s}^{j}-Y_{s}^{j}\right\|}{\sqrt{a_{s}(m)}}=\Theta\left(\frac{E_{s d}(m)}{\sqrt{a_{s}(m)}}\right),
$$

which completes the proof.

3) Delay Analysis for Single-hop Fashion: We provide the delay performance of primary and secondary networks in a single-hop fashion (when $\alpha>\frac{3}{2}$ ) through the following theorem.

Theorem 6. Based on the predefined protocols, the primary and secondary network can achieve the following delays,

$$
D_{p}(n)=\Theta(\log n), D_{s}(m)=\Theta(\log m) .
$$

Proof: According to the analysis of Equation (13), each $\mathrm{S}-\mathrm{D}$ pair is located in a particular cell w.h.p. in this case. Therefore the packet delay is the time for each packet to reach the destination through single-hop transmission. Recall that there are at most $\Theta(\log n)$ nodes in a primary cell, the probability that a particular S-D pair is chosen to transmit data during its active time slot is $P_{\text {Delay, } P}=\Theta\left(\frac{1}{\log n}\right)$. Thus we have $D_{p}(n)=\frac{1}{P_{\text {Delay } P} P}=\Theta(\log n)$. For the secondary network, as the preservation regions will not influence the delay in order sense. Hence, we have $D_{s}(m)=\Theta(\log m)$.

\section{DISCUSSION}

In this section, we will discuss how the heterogeneous distribution of S-D and the cooperation between primary and secondary users affect the throughput and delay scaling law of HCRN. Specifically, $\alpha$ measures the S-D distance. The different ranges of $\alpha$ result in different S-D distances and thus affecting the network performance. We can easily find that by introducing the cooperation between primary and secondary users, the per-node throughput of primary networks can be improved to be $\Theta(1 / \log n)$ compared with $\Theta(1 / \sqrt{n \log n})$ as being a stand-alone network. Further, the number of secondary users required to help improving the PUs throughput can be sharply reduced due to the heterogeneity issue.

\section{A. Near Optimal Performance of Primary Networks}

As defined in the protocols, two-thirds of a secondary time frame are allocated to serve the primary users with the time-sharing strategy. Intuitively, the per-node throughput of primary networks should have varied in accordance with that of secondary networks as the S-D distance changes. However, from Fig.3 we will discover that the primary network can achieve the near optimal per-node throughput of $\Theta\left(\frac{1}{\log n}\right)$, which is independent of $\alpha$. The reason is that the SUs are utilized to help PUs transmit data. By setting appropriate value of $N$, which is defined in Equation (10), the throughput of $\Theta\left(\frac{1}{\log n}\right)$ can always be achieved in whatever ranges of $\alpha$. It is notable that when $\alpha>\frac{3}{2}$ or $\alpha=\frac{3}{2}$, the primary network not only can achieve the near optimal per-node throughput but also can obtain the near optimal delay of $\Theta(\log n)$ and $\Theta(\sqrt{\log n})$ respectively, which is demonstrated in Table II.

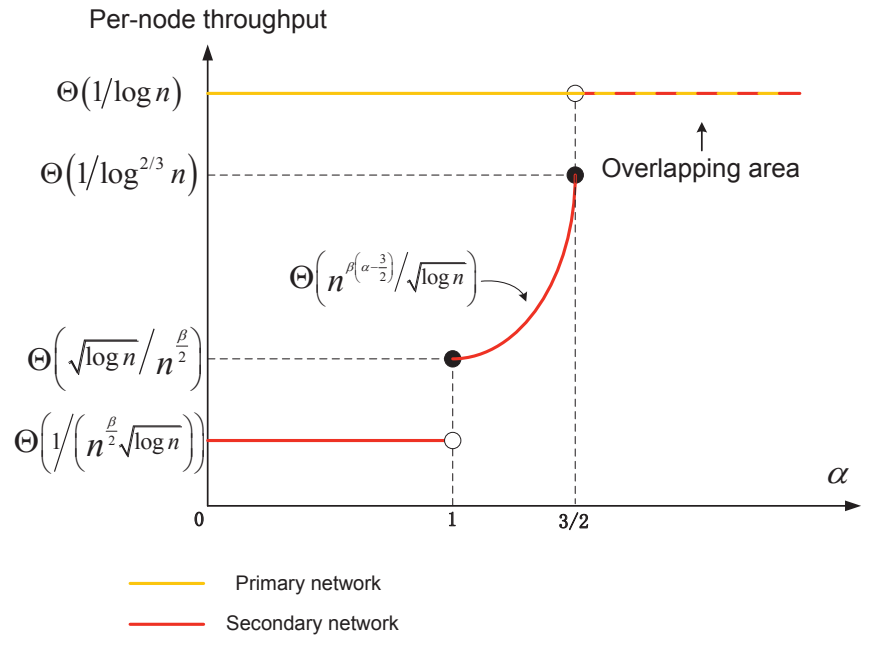

Fig. 3: Per-node throughput scaling laws of primary networks vs secondary networks.

\section{B. Throughput and Delay of Secondary Networks}

Table I illustrates the throughput and delay of secondary networks in different ranges of $\alpha$. According to the protocols previously defined, the proposed time-sharing strategy only incurs a constant penalty on the achievable throughput and delay within the secondary network and thus would not affect the scaling law of secondary networks as being a stand-alone network. As the S-D distance gets shorter with respect to $\alpha$, there is one way in which the interference caused by the concurrent transmission in a local area is reduced, which consequently results in the throughput of secondary networks increasing. In addition, as the S-D distance tends to be shorter, the number of hops which the secondary packets need to pass through is becoming smaller either. Thus, the delay is decreasing. All of the results are demonstrated in Table I.

\section{Economical Deployment of HCRN}

Table II shows the throughput and delay scaling law of primary networks and we also put the required number of SUs on the table. It is easy to find that as the S-D distance tends to be shorter, the number of SUs required to help PUs becomes smaller, where $\delta, \delta^{\prime}, \delta^{\prime \prime}, \varepsilon, \varepsilon^{\prime}$ can be chosen as arbitrary small positive values. Noting that when $0 \leq \alpha<1$, the S-D distance is bounded by $\Theta(1)$ and the number of SUs is $n^{2+\varepsilon^{\prime}}$ which turns to be the same result as that in [14]. So our result is more general compared with that in [14]. More remarkable, as the number of SUs will vary due to the heterogeneous S-D distribution, we can appropriately choose the number of SUs thus making the deployment of HCRN economical in practical scenarios.

\section{CONCLUSION}

In this paper, we study the throughput and delay scaling law of heterogeneous cognitive radio networks (HCRN). We propose the rank based model to illustrate the heterogeneity of S-D distribution and the S-D distance varies with respect 
TABLE I: Throughput and delay of Secondary Networks

\begin{tabular}{|c|c|c|c|}
\hline Range of $\alpha$ & S-D distance & Throughput & Delay \\
\hline \hline$\alpha>\frac{3}{2}$ & $1 / \sqrt{m}$ & $\Theta(1 / \log m)$ & $\Theta(\log m)$ \\
\hline$\alpha=\frac{3}{2}$ & $\log m / \sqrt{m}$ & $\Theta\left(1 / \log ^{\frac{3}{2}} m\right)$ & $\Theta(\sqrt{\log m})$ \\
\hline $1<\alpha<\frac{3}{2}$ & $m^{1-\alpha}$ & $\Theta\left(m^{\alpha-\frac{3}{2}} / \sqrt{\log m}\right)$ & $\Theta\left(m^{\frac{3}{2}-\alpha} / \sqrt{\log m}\right)$ \\
\hline$\alpha=1$ & $1 / \log m$ & $\Theta(\sqrt{\log m} / \sqrt{m})$ & $\Theta\left(\sqrt{m} / \log ^{\frac{3}{2}} m\right)$ \\
\hline $0 \leq \alpha<1$ & 1 & $\Theta(1 / \sqrt{m \log m})$ & $\Theta(\sqrt{m} / \sqrt{\log m})$ \\
\hline
\end{tabular}

TABLE II: Throughput and delay of primary networks and the number of SUs

\begin{tabular}{|c|c|c|c|c|}
\hline Ranges of $\alpha$ and $\beta$ & S-D distance & Throughput & Delay & Number of SUs \\
\hline \hline$\alpha>\frac{3}{2}, \beta>1$ & $1 / \sqrt{n}$ & $\Theta\left(\frac{1}{\log n}\right)$ & $\Theta(\log n)$ & $\Theta\left(n^{1+\delta}\right)$ \\
\hline$\alpha=\frac{3}{2}, \beta>1$ & $\log n / \sqrt{n}$ & $\Theta\left(\frac{1}{\log n}\right)$ & $\Theta(\sqrt{\log n})$ & $\Theta\left(n^{1+\delta^{\prime}}\right)$ \\
\hline $1<\alpha<\frac{3}{2}, \beta \geq 1 /\left(\alpha-\frac{1}{2}\right)$ & $n^{1-\alpha}$ & $\Theta\left(\frac{1}{\log n}\right)$ & $\Theta\left(n^{\beta\left(\frac{3}{2}-\alpha\right)} / \sqrt{\log n}\right)$ & $\Theta\left(n^{\frac{1}{\alpha-\frac{1}{2}}+\delta^{\prime \prime}}\right)$ \\
\hline$\alpha=1, \beta \geq 2$ & $1 / \log n$ & $\Theta\left(\frac{1}{\log n}\right)$ & $\Theta\left(n^{\frac{\beta}{2}} / \log ^{\frac{3}{2}} n\right)$ & $\Theta\left(n^{2+\varepsilon}\right)$ \\
\hline $0 \leq \alpha<1, \beta \geq 2$ & 1 & $\Theta\left(\frac{1}{\log n}\right)$ & $\Theta\left(n^{\frac{\beta}{2}} / \sqrt{\log n}\right)$ & $\Theta\left(n^{2+\varepsilon^{\prime}}\right)$ \\
\hline
\end{tabular}

to $\alpha$. By assuming that the secondary user can pretend to be the primary user, we put forward the corresponding transmission protocols for both primary and secondary networks. In particular, we show that the primary network can achieve the near optimal per-node throughput with the aid of SUs, which is independent of $\alpha$. Meanwhile, the scaling law of secondary networks can be kept the same as being a standalone network. Apparently, the cooperation between PUs and SUs will significantly enhance the transmission of the primary network. We also prove that both the primary and secondary networks can achieve near optimal delay in certain ranges of $\alpha$. Furthermore, we demonstrate that the number of SUs required to help PUs can be sharply reduced due to the heterogeneity of HCRN. This result indicates that the heterogeneity can be utilized to make the network deployment much more economical.

\section{ACKNOWLEDGMENT}

This work is supported by NSF China (No. 61325012, 61271219, 61221001, 61202373); SRF for ROCS by SEM; Shanghai Basic Research Key Project (No. 13510711300, 12JC1405200, 11JC1405100); China Ministry of Education New Century Excellent Talent (No. NCET-10-0580); China Postdoctoral Science Foundation Grant (2012T50417); National Key Project of China (No. 2012ZX03001009); Doctoral Fund of Ministry of Education of China(No. 13005160); SEU SKL project (No. 2012D13) and Open Foundation of State Key Laboratory of Networking and Switching Technology(Beijing University of Posts and Telecommunications) (No. SKLNST-2013-1-16); 973 project (No. 2013CB329006), RGC under the contracts CERG (No. 622410, 622613, HKUST6/CRF/12R), as well as the grant from HuaweiHKUST.

\section{REFERENCES}

[1] P. Gupta, P. R. Kumar, "The Capacity of Wireless Networks," in IEEE Trans. Inform. Theory, vol. 46, no. 2, pp. 388-404, Mar. 2000.

[2] M. Vu, N. Devroye, M. Sharif, and V. Tarokh, "Scaling laws of cognitive networks," in Proc. CrownCom, pp. 2-8, Aug. 2007.

[3] M. Vu, V. Tarokh, "Scaling laws of single-hop cognitive networks," in IEEE Transactions on Wireless Communications, vol. 8, no. 8, pp. 40894097, Aug. 2009.

[4] S.-W. Jeon, N. Devroye, M. Vu, S.-Y. Chung, and V. Tarokh, "Cognitive Networks Achieve Throughput Scaling of a Homogeneous Network," in IEEE Trans. Inform. Theory, vol. 57, no. 8, pp. 5103-5115, Aug. 2011.

[5] C. Yin, L. Gao, and S. Cui, "Scaling Laws for Overlaid Wireless Networks: A Cognitive Radio Network versus a Primary Network," in IEEE/ACM Transactions on Networking, vol. 18, no. 4, pp. 1317-1329, Aug. 2010.

[6] L. Sun and W. Wang, "Understanding Black Holes in Large-scale Cognitive Radio Networks under Generic Failure", in Proceedings of IEEE INFOCOM, Turin, Apr, 2013.

[7] Z. Yang and Y. Liu, "Understanding Node Localizability of Wireless Adhoc Networks", in IEEE Transactions on Mobile Computing, vol. 11, no. 8, pp. 1240-1260, Aug. 2012.

[8] N. M. Tichy, M. L. Tushman, and C. Fombrun, "Social Network Analysis for Organizations," in The Academy of Management Review, vol. 4, no. 4, pp. 507-519, 1979.

[9] Y.-Y. Ahn, S. Han, H. Kwak, S. Moon, and H. Jeong, "Analysis of Topological Characteristics of Huge Online Social Networking Services," in 16th International Conference on World Wide Web, New York, USA, pp. 835-844, 2007.

[10] D. Liben-Nowell, J. Novak, R. Kumar, P. Raghavan, A. Tomkin- s, and R. L. Graham, "Geographic Routing in Social Networks," in Proceedings of the National Academy of Sciences of the United States of America, vol. 102, no. 33, pp. 11623-11628, 2005.

[11] R. Kumar, D. Liben-Nowell, et.al, "Theoretical Analysis of Geographic Routing in Social Networks," in MIT-CSAIL-TR- 2005-040, June 2005.

[12] B. Azimdoost, H. R. Sadjadpour, J. J. Garcia-Luna-Aceves, "The Impact of Social Groups on The Capacity of Wireless Networks," in Proceedings of IEEE Network Science Workshop(NSW), pp. 30-37, 2011.

[13] L. Fu, W. Huang and X. Wang, "Capacity of wireless social networks," Shanghai Jiao Tong University, Tech. Rep., 2011. [Online]. Available: http://iwct.sjtu.edu.cn/Personal/xwang8/paper/social-capacity.pdf

[14] L. Gao, R. Zhang, C. Yin, and S. Cui, "Throughput and Dealy Scaling in Supportive two-tier Networks," in IEEE Jounal on Selected Areas of Communications, vol. 30, no.2, pp. 415-424, 2012

[15] A. E. Gamal, J. Mammen, B. Prabhakar, and D. Shah, "Optimal throughput-daly scaling in wireless networks-part I: the fluid model," in IEEE Trans. Inform. Theory, vol. 52, no.6, pp. 2568-2592, June 2006

[16] M. Franceschetti, O. Dousse, D. N. C. Tse, and P. Thiran, "Closing the Gap in the Capacity of Wireless Networks Via Percolation Theory," in IEEE Trans. Inform. Theory, vol. 53, no. 3, pp. 1009-1018, Mar. 2007. 